\title{
Psychiatric risk peptide of DISC1 inhibits kinase GSK3 $\beta$, Structural investigation using crystallography
}

\author{
N Pujari ${ }^{1}$, S Saundh $^{2}$, S Gagne ${ }^{3}$, A Nambisan ${ }^{4}$, A Leung ${ }^{5}$ \\ ${ }^{1}$ Western College of Veterinary Medicine, University of Saskatchewan, ${ }^{2}$ University of \\ Saskatchewan, Saskatoon, Saskatchewan, ${ }^{3}$ University of Saskatchewan, SASKATOON, \\ Saskatchewan, ${ }^{4}$ Western College of Veterinary Medicine, Saskatoon, Saskatchewan, ${ }^{5}$ Veterinary \\ Biomedical Sciences, University of Saskatchewan \\ narsimha.pujari@usask.ca
}

Disrupted in Schizophrenia 1 (DISC1) is a candidate risk gene in several major mental illnesses, e.g. depression, bipolar disorder, and schizophrenia. The full-length DISC1 protein comprises of 854 amino acids, consisting of many dynamic and disordered regions. DISC1 acts as a scaffold protein. It interacts with a large group of proteins, forming a sizeable protein-protein-interaction network. This interaction network has been implicated in coordination of various stages of the brain development. One of those important interactors is the enzyme, glycogen synthase kinase $3 \beta$ (GSK3 $\beta$ ). As a target for lithium, GSK3 $\beta$ itself is implicated in bipolar disorder. The interaction of DISC1 and GSK3 $\beta$ was discovered at the cross-section of the canonical Wnt $/ \beta$-catenin signalling, which controls the proliferation of neural progenitors. DISC1 specifically inhibits GSK3 $\beta$ 's function in this pathway via a direct physical interaction. The most potent GSK3b inhibitory region has been mapped to a small region in the N-terminus (residue 195-238) of DISC1. This 44-amino acid region (hD1) can inhibit GSK3b in an ATP non-competitive manner. We have obtained different crystal forms of GSK3b bound to hD1 and another peptide that partially shares binding site with $\mathrm{hD} 1$ based on biochemical and biophysical data. As obtaining crystallographic data of these new crystal forms is put on hold due to Covid-19, we are also evaluating whether negative stained electron microscopy can be used to identify large conformational changes of GSK3b induced by $\mathrm{hD} 1$ binding

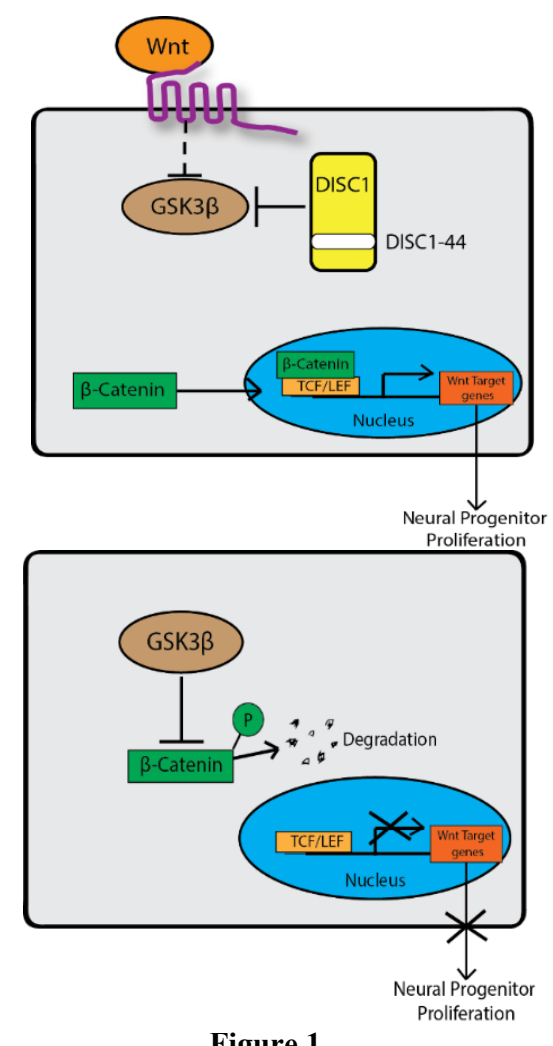

Acta Cryst. (2020). A76, a69 